\title{
Influence of surfactants used in surfactant-polymer flooding on the stability of Gudong crude oil emulsion
}

\author{
Dong Zhaoxia*, Lin Meiqin, Wang Hao and Li Mingyuan \\ Enhanced Oil Recovery Research Center, China University of Petroleum, Beijing 102249, China \\ (c) China University of Petroleum (Beijing) and Springer-Verlag Berlin Heidelberg 2010
}

\begin{abstract}
The influences of an anionic-nonionic composite surfactant and petroleum sulfonate, used in surfactant-polymer flooding in Shengli Gudong oilfield, East China, on the interfacial properties of Gudong crude model oil and synthetic formation water was studied by measuring interfacial tension, interfacial viscoelasticity and Zeta potential. The influence of the surfactants on the stability of Gudong water-in-oil (W/O) and oil-in-water (O/W) emulsions was evaluated by separating water from the W/O emulsion and residual oil in the aqueous phase of the $\mathrm{O} / \mathrm{W}$ emulsion respectively. The results showed that the two kinds of surfactants, namely anionic-nonionic composite surfactant and petroleum sulfonate, are both able to decrease the interfacial tension between the oil phase and the aqueous phase and increase the surface potential of the oil droplets dispersed in the $\mathrm{O} / \mathrm{W}$ emulsion, which can enhance the stability of the $\mathrm{W} / \mathrm{O}$ and $\mathrm{O} / \mathrm{W}$ crude oil emulsions. Compared with petroleum sulfonate, the anionic-nonionic composite surfactant is more interfacially active and able to enhance the strength of the interfacial film between oil and water, hence enhance the stability of the $\mathrm{W} / \mathrm{O}$ and $\mathrm{O} / \mathrm{W}$ emulsions more effectively.
\end{abstract}

Key words: Surfactant, Gudong crude oil, interfacial properties, emulsion, stability

\section{Introduction}

Surfactant-polymer (SP) flooding is an effective technique used in recent years to enhance oil recovery in Shengli Gudong oilfield (East China), which is at the middle-late stage of exploitation. The technique uses partially hydrolyzed polyacrylamide (HPAM) as the polymer. The dissolved HPAM can increase the viscosity of flooding fluid, decrease the mobility ratio of water to oil, and enhance the conformance efficiency. The surfactants can reduce the interfacial tension of water and oil and improve the cleaning efficiency. Therefore, SP flooding can enhance oil recovery greatly.

The bivalent cations dissolved in the reservoir, such as $\mathrm{Ca}^{2+}$ and $\mathrm{Mg}^{2+}$, can precipitate the molecules of HPAM and anion surfactant of petroleum sulfonate, and then reduce the flooding efficiency. To solve this problem, petroleum sulfonate is used as the main surfactant, and an anionic-nonionic composite surfactant is used as cosurfactant in Gudong oilfield. As a result it can reduce the precipitation caused by bivalent cations and decrease the interfacial tension of oil and water by cooperating with petroleum sulfonate, so high SP flooding efficiency can be achieved.

SP flooding is able to increase oil recovery greatly, but this technique is faced with new problems. The production fluid forms stable $\mathrm{W} / \mathrm{O}$ and $\mathrm{O} / \mathrm{W}$ emulsions, and the separation of

*Corresponding author. email: dzx@cup.edu.cn

Received August 22, 2009 crude oil and water is difficult (Kang et al, 1997; Xia et al, 2008; Zhang et al, 2007; Zhang et al, 1998). The stability of the crude oil emulsion are closely related to the interfacial properties between oil and water (Dicharry et al, 2006; Jozef and Mieczyslaw, 1996; Moran et al, 2006; Wang et al, 2008; Xu et al, 2007a). The influence of the polymer used in SP flooding in Gudong oilfield on the stability of crude oil emulsions has been reported (Zong, 2008). In order to study the effect of the surfactants on the stability of the emulsions, the interfacial tension, interfacial shear viscosity and zeta potential between the crude model oil and surfactantsynthesized formation aqueous solution, and the stability of the crude oil emulsions were determined.

\section{Experimental}

\subsection{Materials and preparation of samples}

The crude model oil contains $10 \%$ Gudong crude oil in purified jet fuel. The viscosity of Gudong crude oil is 45.0 $\mathrm{mPa} \cdot \mathrm{s}\left(70{ }^{\circ} \mathrm{C}\right)$, and its density is $0.9218 \mathrm{~g} / \mathrm{cm}^{3}\left(55^{\circ} \mathrm{C}\right)$. The jet fuel was purified by silica adsorption for $48 \mathrm{~h}$ before being used as the dispersion medium for the model oils. The anionic-nonionic composite surfactant (hereinafter referred to as surfactant A) with a content of active component of 58\% (Cao, 2008), and anion surfactant of petroleum sulfonate (i.e. PS) with an active substance content of 50\%, used in experiments were all provided by Gudong oilfield, East China,. The aqueous phase used in the experiments is synthetic formation water (Table 1). 
Table 1 Composition of synthetic formation water of Gudong oilfield

\begin{tabular}{cccccccc}
\hline Ion & $\mathrm{Cl}^{-}$ & $\mathrm{SO}_{4}^{2-}$ & $\mathrm{CO}_{3}^{2-}$ & $\mathrm{HCO}_{3}^{-}$ & $\mathrm{Mg}^{2+}$ & $\mathrm{Ca}^{2+}$ & $\mathrm{Na}^{+}, \mathrm{K}^{+}$ \\
\hline $\begin{array}{c}\text { Concentration } \\
\mathrm{mg} \cdot \mathrm{L}^{-1}\end{array}$ & 7148 & 0 & 0 & 280 & 108 & 632 & 3813 \\
\hline
\end{tabular}

\subsection{Measurement of interfacial properties}

The interfacial tension between the model oils and synthetic formation water with different concentrations of surfactant A was measured by a spinning drop ultra-low interfacial tension meter (Model 500, University of Texas, USA) at $30{ }^{\circ} \mathrm{C}$, and the interfacial tension between them was rather low $\left(<1 \mathrm{mN} \cdot \mathrm{m}^{-1}\right)$. The interfacial tension between the model oils and synthetic formation water with different concentrations of PS was measured by a Wilhelmy interfacial tension meter (DCA-21, Dataphysics, Germany) at $30^{\circ} \mathrm{C}$.

The interfacial shear viscosities were measured using a SVR·S Interfacial Viscoelastic Meter (Kyowa Kagaku Co. Ltd, Japan) at $30^{\circ} \mathrm{C}$.

The zeta potential of the oil droplets dispersed in $\mathrm{O} / \mathrm{W}$ emulsions was measured by a Zetasizer-Nano-ZS (Malvern Instruments Ltd. UK) at $30^{\circ} \mathrm{C}$. A $15 \mathrm{~mW}$ helium-neon laser (wave length in vacuum $\lambda=659 \mathrm{~nm}$ ) was used as the incident beam. The model oil in water, i.e. $\mathrm{O} / \mathrm{W}$ emulsions were prepared by mixing the model oil and synthetic formation water $(\mathrm{O} / \mathrm{W}$ ratio, $1: 100, \mathrm{v} / \mathrm{v})$. The emulsification was carried out by a high speed emulsifier rotating $5 \mathrm{~min}$ at $6,000 \mathrm{rpm}$. The lower liquid of the emulsion, after being left standing for $24 \mathrm{~h}$, was $\mathrm{O} / \mathrm{W}$ emulsion.

\subsection{Measurement of stability of emulsion}

\subsubsection{W/O emulsion}

The model water in oil, i.e. W/O emulsions were prepared by mixing dewatered Gudong crude oil and synthetic formation water containing different concentrations of surfactant $(\mathrm{O} / \mathrm{W}$ ratio, $1: 1, \mathrm{w} / \mathrm{w})$ in a $50 \mathrm{~mL}$ beaker. The emulsification was carried out using a high speed emulsifier rotating for $120 \mathrm{~s}$ at $24,000 \mathrm{rpm}$. The formed emulsion was stored in a stoppered mixing cylinder at $50{ }^{\circ} \mathrm{C}$. The stability of $\mathrm{W} / \mathrm{O}$ emulsions was determined visually by measuring the water volume separated from the emulsions at different times. The volume ratio of separated water to the water added in the emulsion was defined as the separation of water from the emulsion.

\subsection{2 $\mathrm{O} / \mathrm{W}$ emulsion}

The model $\mathrm{O} / \mathrm{W}$ emulsions were prepared by mixing dewatered Gudong crude oil and synthetic formation water containing different concentrations of surfactant $(\mathrm{O} / \mathrm{W}$ ratio, 0.2: $99.8, \mathrm{w} / \mathrm{w})$. The emulsification was carried out using a high speed emulsifier rotating for $120 \mathrm{~s}$ at $6,000 \mathrm{rpm}$. Some of the formed $\mathrm{O} / \mathrm{W}$ emulsion was placed in a $100 \mathrm{~mL}$ graduated test tube and stood for $30 \mathrm{~min}$ at $50{ }^{\circ} \mathrm{C}$. Then 25 $\mathrm{mL}$ of the lower liquid of the emulsion was taken out and put into a separating funnel, and some diluted $\mathrm{HCl}$ (prepared by deionized water/concentrated $\mathrm{HCl}$ ratio of $1: 1, \mathrm{v} / \mathrm{v}$ ) was added to adjust the $\mathrm{pH}$ value of the system to $2-3$, and then $15 \mathrm{~mL}$ petroleum ether was added into the funnel to extract oil. The extraction operation was performed three times. The extraction liquid was placed in a $50 \mathrm{~mL}$ measuring flask and diluted to $50 \mathrm{~mL}$ with petroleum ether. The oil content of the diluted extraction liquid was measured by using an ultraviolet spectrophotometer with a wavelength of $285 \mathrm{~nm}$ at $50^{\circ} \mathrm{C}$.

\section{Results and discussion}

\subsection{Interfacial tension}

The changes of interfacial tension between the model oil and surfactant A, and PS synthetic formation aqueous solution, with time are shown in Fig. 1(a) and Fig. 1 (b). It is clear that the interfacial tension was decreased with the increase of the surfactant concentration, showing that the surfactants are both interfacially active and able to be absorbed at the interface between the model oil and aqueous phase to decrease the interfacial tension. The curves of Fig. 1 (a) and (b) show that the decrease of the interfacial intension between the model oil and surfactant A synthetic formation aqueous solution is greater, with the interface tension below $2.0 \times 10^{-2} \mathrm{mN} \cdot \mathrm{m}^{-1}$, indicating that surfactant $\mathrm{A}$ is more interfacially active.

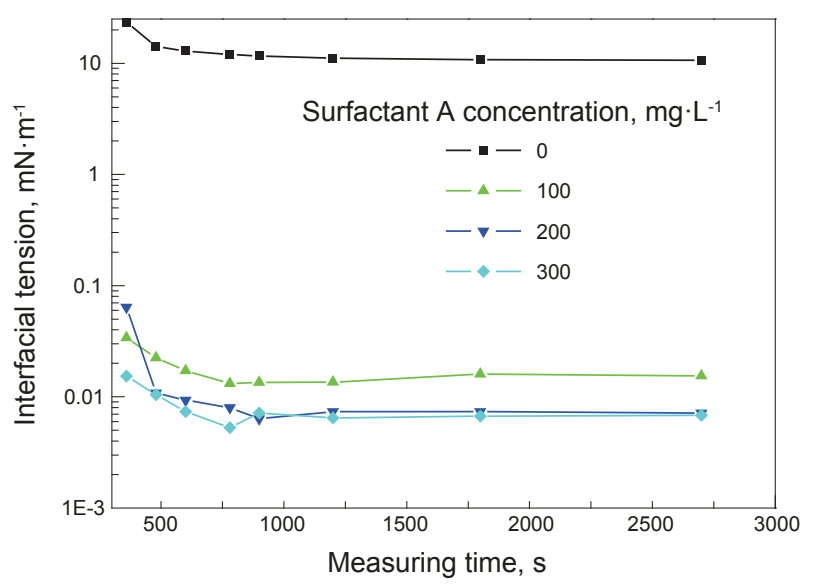

(a) Surfactant A

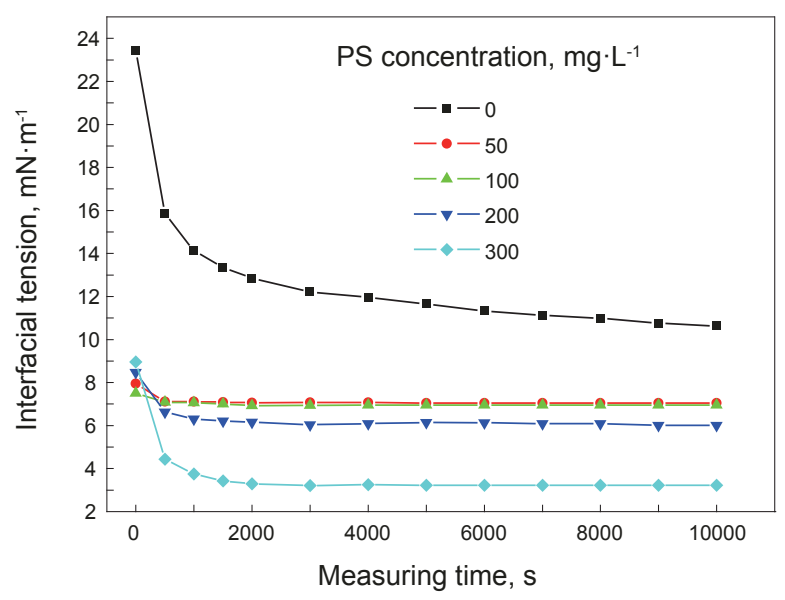

(b) Petroleum sulfonate

Fig. 1 Change of interfacial tension between model oil and surfactant A (a), and PS synthetic formation aqueous solution (b), with time (Oil phase: model oil; aqueous phase: surfactant A or PS synthetic formation aqueous solution; $30{ }^{\circ} \mathrm{C}$ ) 


\subsection{Interfacial shear viscosity}

Fig. 2 shows the changes of the interfacial shear viscosity between the model oil and the aqueous phase with shear rate. The aqueous phase of the system in Fig. 2 (a) was surfactant A synthetic formation aqueous solution. The interfacial shear viscosity of the system increased with the increase of surfactant A concentration. The difference of the interfacial shear viscosity among the systems with different concentrations of surfactant A reduced at high shear rate. The surfactant A molecules were absorbed at the interface between the model oil and the aqueous phase to replace the interfacially active components of crude oil (Cui and Luo, 2009; Ding et al, 1998a; 1998b; Xia et al, 2005; Xu et al, 2007b). The electrostatic repulsion between the surfactant A molecules, in which the non-ionic part was uncharged and small. The surfactant A molecules were able to arrange closely at the interface, as shown in the results of Cao (Cao, 2008). The higher the concentration of surfactant $A$, the closer the arrangement. The close arrangement of the surfactant A molecules at the interface can enhance the strength of the interfacial film between the model oil and the aqueous phase, so the interfacial shear viscosity increased.

The aqueous phase of the system in Fig. 2 (b) was PS

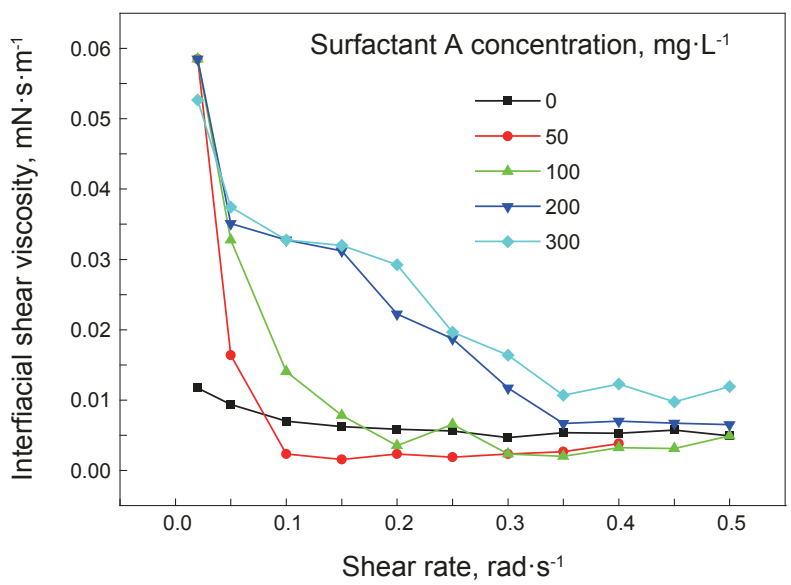

(a) Surfactant A

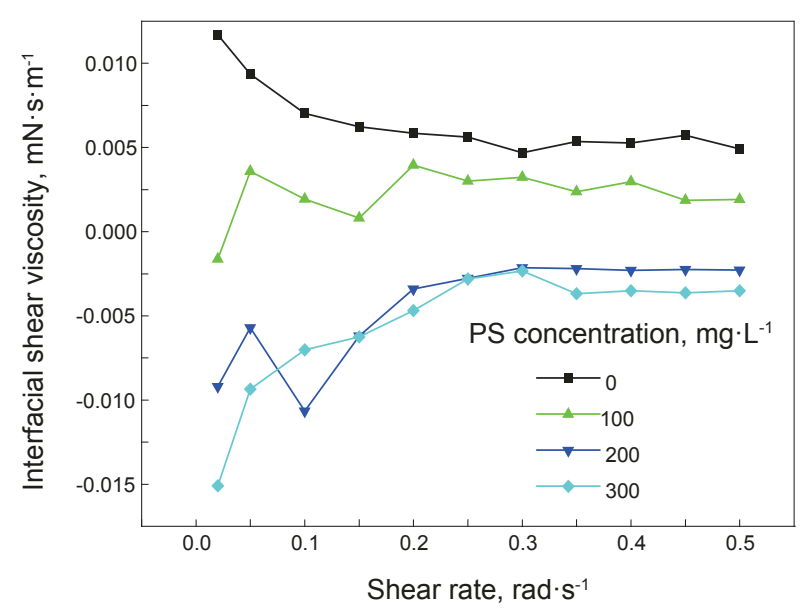

(b) Petroleum sulfonate

Fig. 2 Change of interfacial shear viscosity between the model oil and the aqueous phase with shear rate

(Oil phase: model oil; aqueous phase: surfactant A or PS synthetic formation aqueous solution; $30^{\circ} \mathrm{C}$ ) synthetic formation aqueous solution. The interfacial shear viscosity of the system decreased with the increase of PS concentration. The PS molecules were negatively ionized in the aqueous phase. The electrostatic repulsion between the negative PS molecules was large, and the arrangement of PS molecules at the interface was loose. The loose arrangement of the PS molecules at the interface can reduce the strength of the interfacial film between the model oil and the aqueous phase, so the interfacial shear viscosity was decreased.

\subsection{Zeta potential}

Fig. 3 shows the changes of zeta potential on the crude model oil droplets dispersed in the $\mathrm{O} / \mathrm{W}$ emulsion with surfactant concentration. Before the surfactant or PS was added, the surface of the oil droplets was negative caused by the interfacially active components of the crude oil. With increasing PS concentration the absolute value of zeta potential on the oil droplets was increased. PS is an anionic surfactant which is negative when dissolved in water. With increasing PS concentration, the absorption quantity was also enhanced, which leads to increasing the number of negative charges on the oil droplets, so the absolute value of zeta potential on the oil droplets was increased. With increasing surfactant A concentration the absolute value of zeta potential on the oil droplets was also increased. In spite of weak ionization, surfactant A is more interfacially active and reduce the interface intension between the oil and water dramatically, which makes the oil droplets dispersed in the aqueous phase smaller. The velocity of the oil droplets in the electric field was accelerated, so the absolute value of zeta potential on the oil droplets was increased.

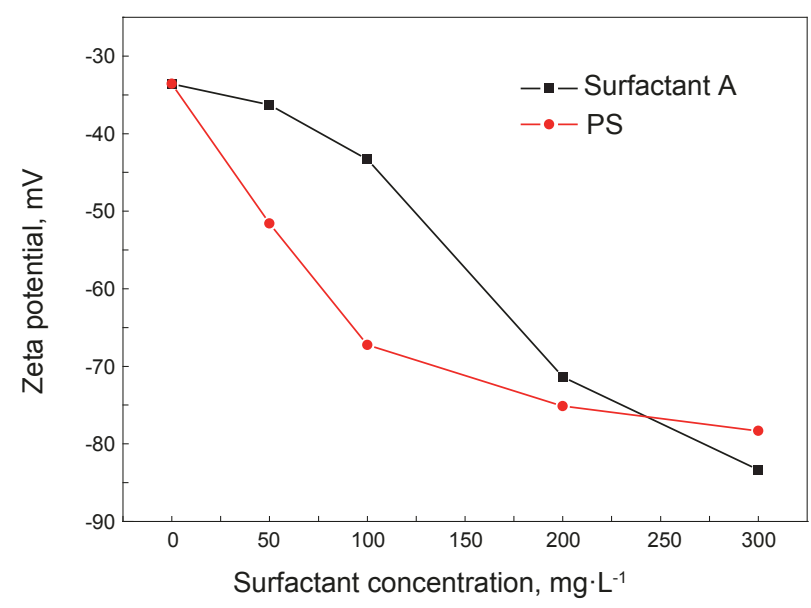

Fig. 3 Change of zeta potential of oil droplets with surfactant concentration

(Oil phase: model oil; aqueous phase: surfactant synthetic formation aqueous solution; $25^{\circ} \mathrm{C}$ )

\subsection{Stability of emulsions}

\subsubsection{W/O emulsion}

The stability of W/O emulsions of crude oil and surfactant synthetic formation aqueous solutions is shown in Fig. 4. With the increase of the surfactant concentration, the amount of water separated from the emulsions decreased for both 
surfactant A and petroleum sulfonate. It indicated that both kinds of surfactants were able to enhance the stability of the $\mathrm{W} / \mathrm{O}$ emulsions. Because surfactant A is more interfacially active than petroleum sulfonate, making the interfacial tension below $2 \times 10^{-2} \mathrm{mN} \cdot \mathrm{m}^{-1}$, it is able to enhance the strength of the interfacial film between the oil phase and aqueous phase, hence the $\mathrm{W} / \mathrm{O}$ emulsion of crude oil and surfactant $\mathrm{A}$ synthetic formation aqueous solution was more stable than that of crude oil and petroleum sulfonate synthetic formation aqueous solution.

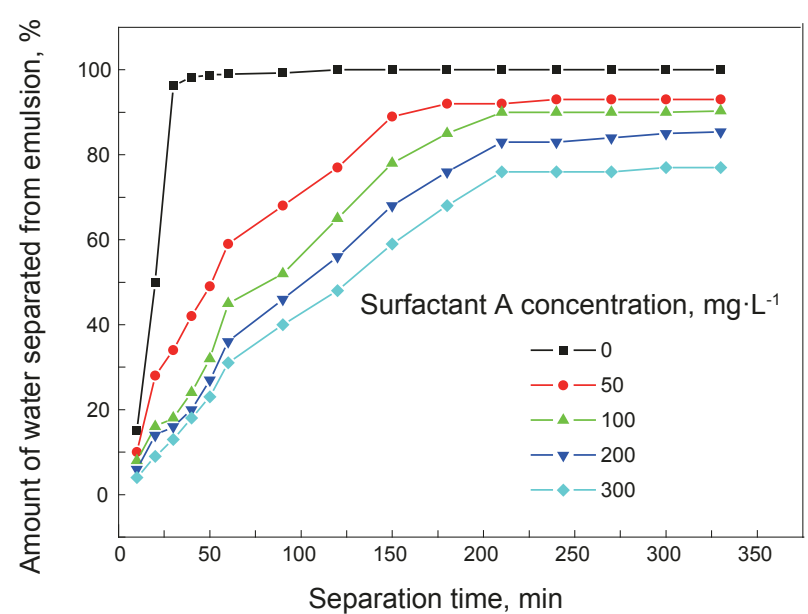

(a) Surfactant A

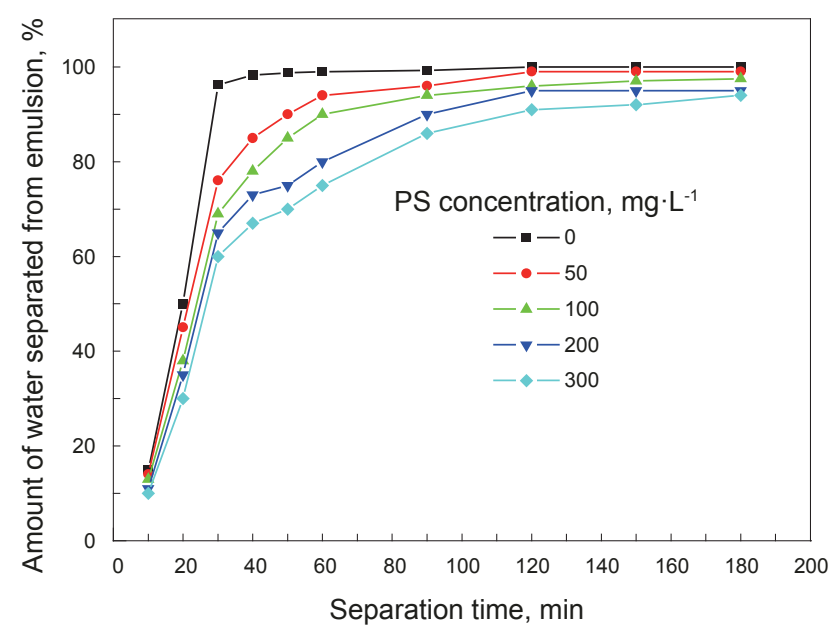

(b) Petroleum sulfonate

Fig. 4 Stability of the W/O emulsion with different surfactant concentrations

(Oil phase: Gudong crude oil; aqueous phase: surfactant synthetic formation aqueous solution; $50{ }^{\circ} \mathrm{C}$ )

\subsection{2 $\mathrm{O} / \mathrm{W}$ emulsion}

Fig. 5 shows the stability of $\mathrm{O} / \mathrm{W}$ emulsions of crude oil and surfactant synthetic formation aqueous solutions. The oil content of the $\mathrm{O} / \mathrm{W}$ emulsions was enhanced with increasing surfactant concentration, indicating that the two kinds of surfactants were able to enhance the stability of the $\mathrm{O} / \mathrm{W}$ emulsions. Surfactant A and petroleum sulfonate both could decrease the interfacial tension and give the surface of the oil droplets in the $\mathrm{O} / \mathrm{W}$ emulsion a high negative potential, which enhanced the stability of the $\mathrm{O} / \mathrm{W}$ emulsions. Because surfactant $\mathrm{A}$ is more interfacially active than petroleum sulfonate and able to increase the strength of the interfacial film between the oil phase and aqueous phase, so the surfactant $\mathrm{A}$-formed $\mathrm{O} / \mathrm{W}$ emulsion was more stable.

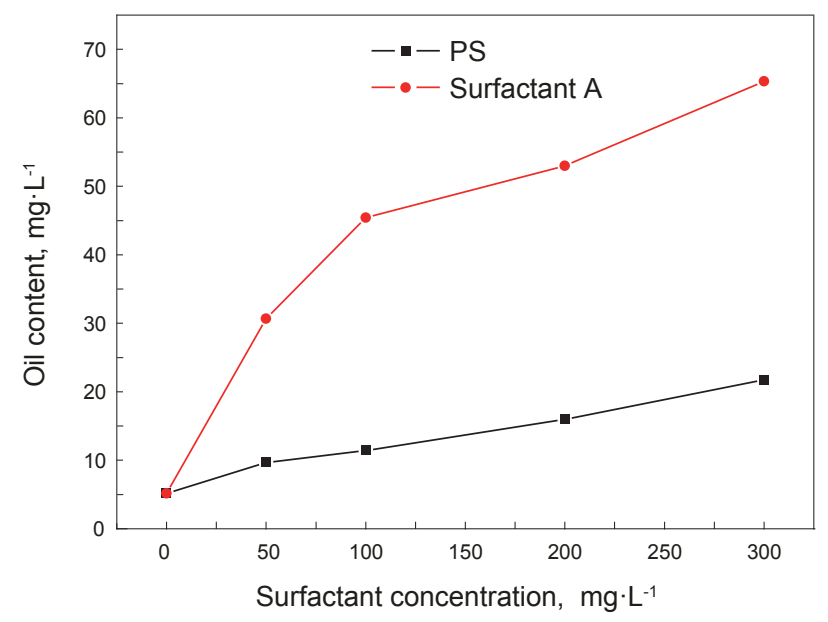

Fig. 5 Stability of the $\mathrm{O} / \mathrm{W}$ emulsion with different surfactants (Oil phase: Gudong crude oil; aqueous phase: surfactant synthetic formation aqueous solution; $50^{\circ} \mathrm{C}$ )

\section{Conclusions}

(1) The anionic-nonionic composite surfactant can decrease the interfacial tension between Gudong crude oil and formation water dramatically, enhance the interfacial shear viscosity and the strength of the interfacial film, and increase the surface negative potential of the oil droplets dispersed in $\mathrm{O} / \mathrm{W}$ emulsion, hence can stabilize the $\mathrm{W} / \mathrm{O}$ and $\mathrm{O} / \mathrm{W}$ emulsions.

(2) Petroleum sulfonate can decrease the interfacial tension between Gudong crude oil and formation water and increase the surface negative potential of the oil droplets dispersed in $\mathrm{O} / \mathrm{W}$ emulsion, hence can make the $\mathrm{W} / \mathrm{O}$ and $\mathrm{O} /$ $\mathrm{W}$ emulsions stable.

(3) Compared with petroleum sulfonate, the anionicnonionic composite surfactant is more interfacially active, which could reduce the interfacial tension of oil and water to below $10^{-2} \mathrm{mN} \cdot \mathrm{m}^{-1}$, and enhance the strength of the interfacial film between the oil and the water, hence enhance the stability of the $\mathrm{W} / \mathrm{O}$ and $\mathrm{O} / \mathrm{W}$ emulsions more.

\section{Acknowledgements}

This work was supported by the National Key Scientific and Technological Projects (2008ZX05011).

\section{References}

Cao X L. Mesoscopic simulation and design on dilute surfactantpolymer system. Acta Petrolei Sinica (petroleum processing section). 2008. 24(6): 682-688 (in Chinese)

Cui B and Luo W. Influences of crude oil fractions on stability of produced water emulsion. Technology \& Development of Chemical Industry. 2009. 38(6): 4-7 (in Chinese) 
Dicharry C, Arla D, Sinquin A, et al. Stability of water/crude oil emulsions based on interfacial dilatational rheology. Journal of Colloid and Interface Science. 2006. 297(2): 785-791

Ding D P, Sun Z C, Xu M Q, et al. Properties of surface film of oil components. Journal of the University of Petroleum, China. 1998a. 22(3): 82-83, 86 (in Chinese)

Ding D P, Sun Z C, Yang G H, et al. Stabilization and breakdown of water in crude oil emulsion: A review. Oilfield Chemistry. 1998b. 15(1): 82-86, 96 (in Chinese)

Jozef S and Mieczysław M. The effect of the $\zeta$ potential on the stability of a non-polar oil-in-water emulsion. Colloid Int. Sci. 1996. 184: 433436

Kang W L, Yue X A and Hu J B. The stability of polymer effect on emulsion and liquid membrane. Acta Petrolei Sinica. 1997. 18(4): 122-125 (in Chinese)

Moran K, Yeung S and Masliyah J. The viscoplastic properties of crude oil-water interface. Chemical Engineering Science. 2006. 61(18): 6016-6028

Wang H Y, Liu A Q and Wen X M. Factors influencing interfacial electric properties of produced water from oilfield. Journal of the University of Petroleum, China. 2008. 32(3): 143-146, 151 (in Chinese)

Xia H F, Wang G, Ma W G, et al. Influence of viscoelasticity and interfacial tension of non-alkali binary compound solution on recovery efficiency of residual oil after water flooding. Acta Petrolei
Sinica. 2008. 29(1): 106-110,115 (in Chinese)

Xia L X, Cao G Y, Lu S X, et al. Influence of asphalthenes and resins on the stability of emulsions. Chemical World. 2005. 9: 521-523, 540 (in Chinese)

Xu M J, Li M Y, Peng B, et al. Effects of strength of interfacial film and zeta potential on oil-in-water emulsion stability. Chinese Journal of Applied Chemistry. 2007a. 24(6): 623-627 (in Chinese)

Xu M J, Li M Y, Peng B, et al. The relationship between stability of water-in-oil emulsion and interfacial shear viscosity of resin and asphaltene. Acta Petroleum Sinica (petroleum progressing section). 2007b. 23(3): 107-110 (in Chinese)

Zhang J Y, Wang X P, Liu H Y, et al. Interfacial rheology investigation of polyacrylamide-surfactant interactions. Colloids Surface. 1998. 132: 9-16

Zhang W, Li M Y, Lin M Q, et al. Effect of interfacial properties of flooding with polymer and surfactant on the stability of emulsion. Petroleum Geology \& Oilfield Development in Daqing. 2007. 26(6): $110-112,118$ (in Chinese)

Zong H. Investigation on oil-water interfacial properties and emulsion stability of polymer flooding produced water in Shengli Oilfield. Applied Chemistry Industry. 2008. 37(10): 1117-1120, 1124 (in Chinese)

(Edited by Zhu Xiuqin) 